\title{
A CONDITION THAT A NORMAL OPERATOR HAVE A CLOSED NUMERICAL RANGE
}

\section{CHING-HWA MENG}

In this paper the following theorem will be proved: $A$ necessary and sufficient condition that the numerical range of a bounded normal operator $T$ in a Hilbert space is closed is that the set of extreme points of the convex closure of the spectrum of $T$ contains no points of the continuous spectrum of $T$.

A few terms should be properly defined. The numerical range of a linear bounded operator $T$ in a Hilbert space is defined as the set of all complex numbers of the form $(T x, x)$ where $x$ is a unit vector in the space. Clearly the numerical range of $T$ contains the point spectrum of $T$. Stone [2] proved that the numerical range of a bounded linear operator $T$ is convex, and that if $T$ is normal then the closure of its numerical range is exactly the convex closure $K(T)$ of the spectrum of $T$. A point $\lambda$ of $K(T)$ is defined as an extreme point of $K(T)$ if no line segment joining any two points of $K(T)$ other than $\lambda$ contains $\lambda$. Denote by $A$ the set of extreme points of $K(T)$. It is easy to see that $A$ is contained in the spectrum of $T$, and that the convex closure of $A$ is exactly $K(T)$. Hence the sufficiency of the condition follows readily from these facts.

The necessity of the condition can be proved by contradiction.

Suppose that there exists a point $a_{0}$ in $A$ such that $a_{0}$ lies in the continuous spectrum of $T$. Now the numerical range of $T$ is closed, and hence $a_{0}$ lies in it. So we can find a unit vector $x$ in the space such that $a_{0}=(T x, x)$. Since $T$ is bounded and normal, it has a resolution of the identity $\left\{E_{\lambda}\right\}$, and $T$ can be represented as an integral with respect to $\left\{E_{\lambda}\right\}$, [1], i.e.,

$$
T=\int_{G} \lambda d E_{\lambda}
$$

where $G$ is any large circle containing the spectrum of $T$ in its interior. Therefore we have:

$$
a_{0}=(T x, x)=\int_{G} \lambda d\left\|F_{\lambda} x\right\|^{2} .
$$

For each $n=1,2,3, \cdots$, consider the open circle $D_{n}$ about the

Received by the editors April 6, 1956. 
point $a_{0}$ with radius $1 / 2^{n}$, and denote by $X_{n}(\lambda)$ the characteristic function of $D_{n}$, i.e., $X_{n}(\lambda)=1$ for $\lambda$ in $D_{n}$, and $=0$ otherwise. Since every Borel set is measurable with respect to $\left\{E_{\lambda}\right\},[1]$, for each $n=1,2,3, \cdots$, we have

$$
\begin{aligned}
I & =\int_{G} d E_{\lambda} \\
& =\int_{G} X_{n}(\lambda) d E_{\lambda}+\int_{G}\left[1-X_{n}(\lambda)\right] d E_{\lambda} \\
& =\int_{G_{n}} X_{n}(\lambda) d E_{\lambda}+\int_{R_{n}}\left[1-X_{n}(\lambda)\right] d E_{\lambda},
\end{aligned}
$$

where $G_{n}$ is any convex region containing $D_{n}$ in its interior, and $R_{n}$ is any region containing in its interior the set of the spectrum of $T$ less $D_{n}$; in particular, one can choose $R_{n}$ such that $a_{0}$ is at a positive distance outside of $R_{n}$ and $R_{n}$ is convex. This can be done because $a_{0}$ is an extreme point of $K(T)$. Let for each $n=1,2,3, \cdots$,

$$
F_{1, n}=\int_{G_{n}} X_{n}(\lambda) d E_{\lambda}, \quad F_{2, n}=\int_{R_{n}}\left[1-X_{n}(\lambda)\right] d E_{\lambda},
$$

then $F_{1, n}$ and $F_{2, n}$ are projection operators and are orthogonal to each other. It is claimed that for each $n=1,2,3, \cdots, F_{2, n} x=\theta$. Suppose not, then let $c_{i, n}=\left\|F_{i, n} x\right\|^{2}$ for $i=1,2$. So we have for some $n$, $c_{2, n}>0$, and

$$
1=\left\|F_{1, n} x+F_{2, n} x\right\|^{2}=\left\|F_{1, n} x\right\|^{2}+\left\|F_{2, n} x\right\|^{2}=c_{1, n}+c_{2, n} .
$$

Hence $0 \leqq c_{1, n}<1$ and $0<c_{2, n} \leqq 1$. Since the regions $G_{n}$ and $R_{n}$ are convex, we may apply the mean value theorem and deduce that

$$
\begin{aligned}
a_{0} & =(T x, x)=\int_{G} \lambda d\left\|E_{\lambda} x\right\|^{2} \\
& =\int_{G_{n}} \lambda X_{n}(\lambda) d\left\|E_{\lambda} x\right\|^{2}+\int_{R} \lambda\left[1-X_{n}(\lambda)\right] d\left\|E_{\lambda} x\right\|^{2} \\
& =\lambda_{1, n} c_{1, n}+\lambda_{2, n} c_{2, n}
\end{aligned}
$$

where $\lambda_{1, n}$ lies in $G_{n}$, and $\lambda_{2, n}$ lies in $R_{n}$. Since $R_{n}$ is so chosen that it is at a positive distance away from $a_{0}$, therefore $\lambda_{2, n}$ is not equal to $a_{0}$ which implies that $0<c_{1, n}<1,0<c_{2, n}<1$, and $\lambda_{1, n} \neq a_{0}$. Hence both $F_{1, n} x$ and $F_{2, n} x$ are not zero vectors. Consider the unit vector $y_{2, n}$ $=F_{2, n} x /\left\|F_{2, n} x\right\| ;$ then we have 


$$
\begin{aligned}
\left(T y_{2, n}, y_{2, n}\right) & =\int_{G} \lambda d\left\|E_{\lambda} y_{2, n}\right\|^{2} \\
& =\frac{1}{\left\|F_{2, n} x\right\|^{2}} \int_{G} \lambda d\left\|E_{\lambda} F_{2, n} x\right\|^{2} \\
& =\frac{1}{c_{2, n}} \int_{G} \lambda d \int_{R_{n}}\left[1-X_{n}(\mu)\right] d\left\|E_{\lambda} E_{\mu} x\right\|^{2} \\
& =\frac{1}{c_{2 n}} \int_{R_{n}} \lambda\left[1-X_{n}(\lambda)\right] d\left\|E_{\lambda} x\right\|^{2}=\lambda_{2, n} .
\end{aligned}
$$

Hence $\lambda_{2, n}$ lies in the numerical range of $T$. Similarly, $\lambda_{1, n}$ lies in the numerical range of $T$. Now by hypothesis the numerical range is closed, and $T$ is normal, and consequently the numerical range is exactly $K(T)$. However, we have

$$
a_{0}=c_{1, n} \lambda_{1, n}+c_{2, n} \lambda_{2, n}
$$

with $c_{1, n}+c_{2, n}=1,0<c_{1, n}, c_{2, n}<1$, and $\lambda_{1, n} \neq a_{0} \neq \lambda_{2, n}$. Furthermore $a_{0}$ is an extreme point of $K(T)$. So this is a contradiction.

Therefore we conclude that $F_{2, n} x$ is a zero vector for all $n=1,2$, $3, \cdots$, which implies that $F_{1, n} x=x$ for all $n=1,2,3, \cdots$. Now for all $n=1,2,3, \cdots$,

$$
\begin{aligned}
T x & =\int_{G} \lambda d E_{\lambda} x=\int_{G} \lambda d E_{\lambda} F_{1, n} x \\
& =\int_{G} \lambda d \int_{G_{n}} X_{n}(\mu) d E_{\lambda} E_{\mu} x \\
& =\int_{G_{n}} \lambda X_{n}(\lambda) d E_{\lambda} x,
\end{aligned}
$$

and

$$
\begin{aligned}
\left\|T x-a_{0} x\right\| & =\left\|\int_{G_{n}} \lambda X_{n}(\lambda) d E_{\lambda} x-a_{0} \int_{G_{n}} X_{n}(\lambda) d E_{\lambda} x\right\| \\
& =\left\|\int_{G_{n}}\left(\lambda-a_{0}\right) X_{n}(\lambda) d E_{\lambda} x\right\| \\
& \leqq \max _{\lambda \in D_{n}}\left|\lambda-a_{0}\right| \leqq \frac{1}{2^{n}} \rightarrow 0
\end{aligned}
$$

as $n \rightarrow \infty$. Therefore $T x=a_{0} x$ which implies that $a_{0}$ lies in the point spectrum of $T$. However the point spectrum and the continuous spec- 
trum of $T$ are mutually exclusive, and consequently we have a contradiction. Hence the theorem is proved. The following corollary is an immediate consequence of the theorem:

The numerical range of a unitary operator in a Hilbert space is closed if and only if the spectrum of the unitary operator consists entirely of the point spectrum.

\section{REFERENCES}

1. B. Sz.Nagy, Spektraldarstellung linearer Transformationen des Hilbertschen Raumes, Ergebnisse der Mathematik vol. 5, Berlin, 1942.

2. M. H. Stone, Linear transformations in Hilbert space and their applications to analysis, Amer. Math. Soc. Colloquium Publications, vol. 15, New York, 1932.

Sacramento State College

\section{ON POSTULATES FOR GENERAL QUANTUM MECHANICS}

\section{B. LOWDENSLAGER ${ }^{1}$}

A collection of postulates for the observable quantities in a general quantum mechanical system has been given by Segal, [4]. In this system, the observables form a real Banach space $S$ which is partially ordered by a closed convex positive cone $P$, and which has a unit element $e$ interior to the positive cone, all satisfying $P \cap(e-P)$ $=S_{1}(e) \cap S_{1}(0)$, where $S_{r}(x)$ is the solid sphere of radius $r$, center $x$. Alternatively, $S$ is a partially ordered linear space with an element $e>0$ which is a Banach space under the norm $\|x\|=\min \{\lambda:-\lambda e$ $\leqq x \leqq \lambda e\}$, where the minimum is attained. Let us call such a space a partially ordered Banach space with unit. We shall show that any such Banach space $S$ with order unit $e$ can be given the algebraic structure postulated by Segal, in possibly different ways. In this proof it is very useful to have an alternative formulation of Segal's postulates stated in terms of the partial ordering alone. We are then able to give examples of such systems with various pathologies and to classify the three dimensional ones. Finally we shall give a necessary and sufficient condition that a system of operators satisfying these postulates be a $C^{*}$ special Jordan algebra: the system of hermitean elements of a $C^{*}$ algebra.

Definition. A spectral function $L$ on a partially ordered Banach space $S$ with order unit $e$ is a function assigning to each point $x \in S$ a

Received by the editors February 27, 1956.

1 This work was done under contract Nonr-222(37) with the O.N.R. 